\title{
Prevención del Dolor en Recién Nacidos de Término: Estudio Aleatorizado Sobre Tres Métodos
}

\author{
Dr. GERMÁN BONETTO ${ }^{1}$, Lic. Enf. ESTELA SALVATICO ${ }^{1}$, Enf. Prof. NATALIA VARELA ${ }^{1}$, \\ Lic. Enf. CRISTINA COMETTO' ${ }^{1}$, Lic. Enf. PATRICIA F. GÓMEZ ${ }^{1}$ y Dr. BERNARDO CALVO ${ }^{1}$ \\ 1. Servicio de Pediatría. Sanatorio Allende. Córdoba. Argentina.
}

\begin{abstract}
"La ciencia no nos ofrece verdades absolutas, pero nos da respuestas con cierto grado de certeza."

MARK MORRIS

BMJ 2000;320:1002
\end{abstract}

\begin{abstract}
Pain prevention in term neonates: randomised trial for three methods

Introduction: Every newborn infant is screened for hypothyroidism and phenilketonuria by blood sampling, during the first week of life, but there is not a simple and efficient method to reduce pain during the procedure. Objectives: Prospective randomized trial, to assess if the administration of oral glucose, paracetamol or EMLA, given individually, can reduce the pain caused in newborns by heel prick, in an outpatient setting. Methods: Double-blind study in which seventy six healthy newborns at term were randomly assigned to receive placebo, oral glucose, EMLA in the heel, or oral paracetamol. Heel prick was performed to get a blood sample, and pain was measured by two independent observers, using two scales (NIPS and PIPP). Results: NIPS $<4$ : placebo $(9 / 19=47 \%)$, glucose $(16 / 19=84 \%)$, paracetamol $(8 / 19=42 \%$ and EMLA $(12 / 19$ $=63 \%)$; PIPP < 8: placebo $(9 / 19=47 \%)$, oral glucose $(12 / 19=63 \%)$, paracetamol $(5 / 19=26 \%)$ and EMLA $(8 / 19=42 \%)$. With the use of oral glucose we found RAR: 0.37 (IC 95\% 0.09-0.64), RRR: 44\% (IC 95\% 6-67\%), NNT: 2.7 (IC 95\% 1.5-11). Conclusions: The best results were obtained with the use of oral glucose, being statistically significant with only one of the scales. The administration of paracetamol or EMLA did not reduce pain. Other complementary and/or combined methods, added to oral glucose, should be considered daily to diminish this painful experience in thousands of children.

(Key words: Neonate, analgesia, heel punction, outpatient setting).

Arch Argent Pediatr 2008; 106 (5): 392-396/392
\end{abstract}

Correspondencia a:

Dr. Bernardo Calvo.

E-mail: bcalvo@sanatorioallende.com 


\section{RESUMEN}

Introducción: Por norma legal, a todos los recién nacidos se les debe extraer sangre durante los primeros días de vida, para la pesquisa neonatal de hipotiroidismo congénito y fenilcetonuria. El procedimiento de extracción supone cierto dolor para el neonato y aún no existe un método sencillo y eficaz para disminuirlo. Objetivos: Evaluar si la administración de glucosa, paracetamol o EMLA, disminuyen individualmente el dolor en recién nacidos, provocado por punción de talón, en un ambiente ambulatorio. Material y Métodos: Estudio prospectivo y aleatorizado, a doble ciego, en el cual setenta y seis recién nacidos de término y sanos fueron asignados a recibir placebo, glucosa por vía oral, EMLA en talón o paracetamol por vía oral. Se realizó punción de talón para extraer sangre y dos observadores independientes midieron el dolor con dos escalas (NIPS y PIPP). La variable de resultado fue la cantidad de niños con valores de dolor por debajo de las cifras consideradas dolor moderado en cada grupo. Resultados: NIPS $<4$ : placebo $(9 / 19=47 \%)$, glucosa $(16 / 19=84 \%)$, paracetamol $(8 / 19=42 \%)$ y EMLA $(12 / 19=63 \%)$. PIPP $<8$ : placebo $(9 / 19=47 \%)$, glucosa $(12 / 19=63 \%)$, paracetamol $(5 / 19=26 \%)$ y EMLA $(8 / 19=42 \%)$. Con el uso de glucosa oral, encontramos: RAR: 0,37 (IC 95\%: 0,09-0,64), RRR: 44\% (IC 95\%: 6-67), NNT: 2,7 (IC 95\% 1,5-11). Conclusiones: Con el uso de la solución glucosada por vía oral se obtuvieron los mejores resultados. Sólo con una de las escalas utilizadas fueron estadísticamente significativos. Con paracetamol y EMLA, no observamos disminución del dolor. Deberán considerarse otros métodos complementarios y combinados, junto con la glucosa, para aliviar esta experiencia dolorosa a miles de niños diariamente.

(Palabras clave: Recién nacidos, analgesia, punción de talón, ambiente ambulatorio). Arch Argent Pediatr 2008; 106 (5): 392-396/392

ESTE TRABAJO LO PUEDE ENCONTRAR EN EXTENSO EN WWW.SciELO.ORG 